Pacific Journal of Mathematics

TWO SOLVABILITY THEOREMS 


\section{TWO SOLVABILITY THEOREMS}

\section{M. ISAACS}

\section{In this paper we prove two theorems which have certain similarities.}

Theorem I. Let $G$ be a group with a cyclic $S_{p}$ subgroup $P$ such that every $p^{\prime}$-subgroup of $G$ is abelian. Then either $G$ has a normal $p$-complement or else $P \Delta G$.

Theorem II. Let $G$ be a group and let $p \neq 2$ and $q$ be primes dividing $|G|$. Suppose for every $H<G$ which is not a $q$-group or a $q^{\prime}$-group that $p \| H \mid$. If $q^{a}$ is the $q$-part of $|G|$ and $p>q^{a}-1$ or if $p=q^{a}-1$ and an $S_{p}$ of $G$ is abelian then no primes but $p$ and $q$ divide $|G|$.

Both theorems are proved by studying minimal counter-examples and in both cases contradictions are obtained for $p>3$ without the use of character theory. When $p=3$ both minimal counterexamples satisfy the hypotheses of the same character theoretic proposition which is actually a special case of Theorem II, and this yields the desired contradictions.

Both theorems imply that the respective groups in question are solvable. In the first case the Schur-Zassenhaus Theorem (see 9.3.6 of [5]) is used and in the second case Burnside's $p^{i} q^{j}$ theorem (see 12.3.3 of [5]) yields the solvability.

1. In this section we prove the character theoretic proposition which is a special case of Theorem II and which is used to prove both of our main results. We begin by giving a lemma which is a restatement of some of the restlts of $\S$ II of [1].

LEMma 1. (Brauer-Fowler) Let $G$ be a group of even order which has only one class of involutions $K_{0}$ with $m=\left|K_{0}\right|$. Let $K_{i}, 1 \leqq i \leqq r$ be the remaining nonidentity real classes in $G$. Then

$$
m^{2}=u m+\sum_{i=1}^{r} v_{i}\left|K_{i}\right|
$$

where $u$ is the number of involutions in the centralizer of an involution and $v_{i}$ is the number of involutions which transform $x$ to $x^{-1}$ when $x \in K_{i}$.

Proposition. Let $G$ be a group with an abelian $S_{3}$ subgroup $P$ with the properties

(1) $\left|\mathfrak{N}_{G}(P)\right|=4|P|,\left|\mathfrak{\Im}_{\theta}(P)\right|=2|P|$, 
(2) $\mathfrak{V}_{G}(P)$ is a T.I. set and

(3) if $H<G$ has even order then $|H| \mid(4|P|)$. Then $G$ is not simple.

Proof. Suppose $G$ is simple. It is clear that the order of an $S_{2}$ of $G$ is 4 and thus by Burnside's theorem it must be elementary and all of its involutions are conjugate in its normalizer. Put

$$
S=\mathfrak{S}_{G}(P)=P \times\langle s\rangle \text { and } N=\mathfrak{N}_{G}(P)=S\langle t\rangle,
$$

where $s$ and $t$ are commuting involutions. Since $G$ is simple and $P$ is abelian, we have $P \bigcap Z(\Re(P))=1$ by 13.5 .5 of [5] and thus $\mathfrak{S}_{P}(t)=1$ and $t$ acts on $P$ with no nontrivial fixed points. Therefore $t$ transforms every element of $P$ and thus also of $S$ into its inverse. Clearly $S \triangle N$ and $P \triangle \mathfrak{R}_{G}(S)$ and thus $N=\mathfrak{R}_{G}(S)$. If two elements of $S$ are conjugate in $G$ they are conjugate in $N$ since $S$ is a $T$. I. set and if they are distinct they are inverses. Since the only elements of $S$ equal to their inverses are $s$ and 1 , the remaining $2|P|-2$ elements of $S$ span $|P|-1$ classes of $G$.

If $y \neq 1$ is a real element of $G$ which is not an involution then $\mathfrak{N}_{G}(\langle y\rangle)<G$ has even order and thus $y$ has order divisible by 3 and centralizes some element of order 3. By taking conjugates we may suppose that this element is in $P$ and therefore $y \in N$. Since no element of $N-S$ centralizes any element $\neq 1$ in $P$, we conclude that $y \in S$. Therefore the $|P|-1$ classes spanned be the nonself-inverse elements of $S$ are the classes $K_{i}$ of the lemma and $r=|P|-1$.

Since $\mathfrak{C}_{G}(s) \supseteqq N$ and $\left|\mathfrak{C}_{G}(s)\right| \mid(4|P|)$ we must have $\mathfrak{E}(s)=N$. Every element of $N-S$ is an involution and therefore in the lemma we have $u=2|P|+1$. Since $\subseteq(s)=N, m=[G: N]=|G| / 4|P|$. If $x \in S$ and $x \neq 1, s$ then $\mathbb{E}_{\theta}(x)=S$ and $\left|K_{i}\right|=[G: S]=2 m$. Finally, the only involutions transforming $x$ to $x^{-1}$ are the elements of $N-S$ and hence each $v_{i}=2|P|$ and the lemma yields

$$
m^{2}=(2|P|+1) m+(|P|-1)(2|P|)(2 m)
$$

and therefore $m=4|P|^{2}-2|P|+1$ and $|G|=4|P| m$.

Now $G$ has $|P|+1$ real classes and thus by Theorem 12.4 of [4] it has $|P|$ irreducible, nonprincipal real valued characters, $\chi_{i}$, $1 \leqq i \leqq|P|$. Since $G$ has $m$ involutions,

$$
m=\sum_{i=1}^{|P|} \chi_{i}(1) \varepsilon_{i}
$$

where $\varepsilon_{i}= \pm 1$ by Theorem 3.6 of [4]. Therofore $m \leqq \sum_{i=1}^{|P|} \chi_{i}(1)$ and we have 


$$
m^{2} \leqq\left[\sum_{i=1}^{|P|} \chi_{i}(1)\right]^{2} \leqq|P| \sum_{i=1}^{|P|} \chi_{i}(1)^{2}=|P|\left[|G|-\sum \psi_{j}(1)^{2}-1\right]
$$

where the $\psi_{j}$ are the irreducible nonreal valued characters. Thus

$$
|P| \sum \psi_{j}(1)^{2} \leqq|P|(|G|-1)-m^{2} \leqq m\left(4|P|^{2}-m\right)
$$

since $|G|=4|P| m$. Since $4|P|^{2}-m=2|P|-1<2|P|$, we have $\sum \psi_{j}(1)^{2}<2 m$. Because $G$ contains elements of order prime to 6 , not every class of $G$ is real and thus some $\psi$ exists with $\psi \neq \bar{\psi}$ and hence $\psi(1)^{2}<m$.

Now $[N: S]=2$ and $S$ is abelian and thus all nonlinear irreducible characters of $N$ have degree 2 . Since $t$ acts without fixed points on $P$, it is clear that $N^{\prime}=P$ and $N$ has exactly 4 linear characters and thus has $|P|-1$ distinct irredudcible characters of degree 2, say $\lambda_{1}, \cdots, \lambda_{|P|-1}$. Since $[N: S]=2$ and $\lambda_{i} \mid S$ is reducible, it follows that $\lambda_{i}$ vanishes on $N-S$ and we may apply Theorem 38.16 of [3] since $S$ is a $T$. $I$. set. Therefore $G$ has irreducible characters

$$
\zeta_{1}, \zeta_{2}, \cdots, \zeta_{|P|-1}
$$

and there is $\varepsilon= \pm 1$ with $\lambda_{i}^{G}-\lambda_{j}^{G}=\varepsilon\left(\zeta_{i}-\zeta_{j}\right)$. Since each $\lambda_{i}^{G}$ is real valued, the same is true of the $\zeta_{i}$ and thus we have the inner pro$\operatorname{duct}\left[\psi,\left(\lambda_{i}^{G}-\lambda_{j}^{G}\right)\right]=0$. Therefore

$$
\left[\psi, \lambda_{i}^{G}\right]=\left[\psi, \lambda_{j}^{G}\right]
$$

and by Frobenius Reciprocity, $\left[\psi \mid N, \lambda_{i}\right]=\left[\psi \mid N, \lambda_{j}\right]$. We conclude that the multiplicities of each $\lambda_{i}$ in $\psi \mid N$ are equal. Since $\psi$ is faithful and $N$ is nonabelian, $\psi \mid N$ has some nonlinear constituent and thus this common multiplicity is $\geqq 1$ and therefore $\psi(1) \geqq 2(|P|-1)$. Since $\psi(1)^{2}<m<4|P|^{2}$, we have $\psi(1)<2|P|$ and thus

$$
\psi(1)=2|P|-2 \text { or } 2|P|-1 \text {. }
$$

Let $q$ be the largest prime divisor of $\psi(1)$. If $q=2$ then since $\psi(1)|| G \mid$ we must have $\psi(1)=4=2|P|-2$ and $|P|=3$. In this situation $m=31$ and $|G|=12 \cdot 31$ and since no simple group can have this order, we have a contradiction. Thus $q \neq 2$ and since $3 \||P|$, $q>3$. Since $q|| G \mid$ we must have $q \mid m$ and $4|P|^{2}-2|P|+1 \equiv 0$ $\bmod q$. Since $2|P| \equiv 1$ or $2 \bmod q$, we have $4|P|^{2}-2|P|+1 \equiv 1$ or $3 \bmod q$. Since $q>3$ this is our final contradiction.

2. In this section we prove the first of our main results. We begin with a lemma.

Lemma 2. Let $H$ be an abelian group with a collection of proper subgroups $\left\{K_{i}\right\}$ such that $H=\bigcup K_{i}$ and $K_{i} \cap K_{j}=1$ if $i \neq j$. Then 
$H$ is an elementary abelian p-group for some prime $p$.

Proof. If $x, y \in H^{\sharp}$ have different orders $m$ and $n$ respectively, with $m>n$, choose $K_{i}$ with $x \in K_{i}$. Then $1 \neq(x y)^{n}=x^{n} \in K_{i}$. If $x y \in K_{j}$ then $(x y)^{n} \in K_{i} \cap K_{j}$ and therefore $i=j$ and $x y \in K_{i}$. Thus $y \in K_{i}$. If $z \in H^{\sharp}$ is arbitrary then the order of $z$ is different from at least one of $m$ and $n$ and thus $z \in K_{i}$. Thus $K_{i}=H$ and this contradiction shows that all elements of $H^{\#}$ have equal orders and the result follows.

THEOREM I. Let $G$ be a group with a cyclic $S_{p}$ subgroup $P$ such that every $p^{\prime}$-subgroup of $G$ is abelian. Then $G$ has a normal $p$ complement or else $P \triangle G$.

Proof. Suppose the theorem is false and let $G$ be a minimal counterexample. Let $N=\mathfrak{N}_{G}(P)$ and let $K$ be an $S_{p^{\prime}}$ (p-complement) of $N$ whose existence is guaranteed by the Schur-Zassenhaus Theorem (9.3.6 of [5]). If any element $x \in K$ centralizes a nonidentity element of $P$, then because $P$ is cyclic, $x$ centralizes all of $P$. (See for instance 20.1 of [4]).

Every proper subgroup of $G$ satisfies the hypotheses and thus has either a normal $S_{p}$ or $S_{p^{\prime}}$. If $L \triangle G$ and $p \nmid|L|$ then $G / L$ satisfies the hypotheses and does not have a normal $S_{p^{\prime}}$ and therefore if $L>1, P L \triangle G$. By Burnside's theorem, $K \triangle N$ and thus $N L$ does not have a normal $S_{p^{\prime}}$ and if $N L<G, L$ normalizes $P$ and $P$ is characteristic in $P L$ and thus is normal in $G$. This contradiction shows that $N L=G$. Now put $M=\bigcap_{x \in G} N^{x} \triangle G$. Since $x=u v$ for some $u \in N$ and $v \in L$ we have $N^{x}=N^{u v}=N^{v} \supseteqq K^{v}$. However $K L$ is a $p^{\prime}$-subgroup and thus is abelian and $K^{v}=K$. Since $x$ was arbitrary, $M \supseteqq K$ and thus $M \supseteqq K^{u}$ for all $u \in N$. Since $K$ is an $S_{p^{\prime}}$ of the solvable group $M$ we may conclude that $K^{u}$ is conjugate to $K$ in $M$ by P. Hall's theorem (9.3.10 of [5]) and therefore there exists $w \in M$ with $u w^{-1} \in \mathfrak{N}_{N}(K)$. If $\mathfrak{N}_{N}(K)>K$ then $\mathfrak{R}_{P}(K)>1$. This group is normalized and thus centralized by $K$ and thus all of $P$ is also. This contradiction shows that $\mathfrak{N}_{N}(K)=K, u w^{-1} \in K$, and thus $N=M K$. Since $p \nmid|K|, P \subseteq M$ and we have $M=N$ and thus all $N^{x}$ are equal and $N \triangle G$. Thus $P \triangle G$ and we have a contradiction. Our assumption on the existence of $L$ is therefore invalid and $\mathfrak{D}_{p^{\prime}}(G)=1$.

If $P_{0} \triangle G$ is a $p$-group, put $C=\mathfrak{\Im}_{G}\left(P_{0}\right) \triangle G$. If $C=G$ then $K$ centralizes $P_{0}$ and therefore $K$ centralizes all of $P$ and we have a contradiction. Thus $C<G$ and since $P \subseteq C, C$ does not have a normal $S_{p}$. Therefore $C$ is not a $p$-group and has a normal $S_{p^{\prime}}$ and this contradicts $\mathfrak{D}_{p^{\prime}}(G)=1$ and we conclude that $\mathfrak{D}_{p}(G)=1$. If $L \neq 1$ 
is any proper normal subgroup of $G$ then either an $S_{p}$ or an $S_{p^{\prime}}$ of $L$ is normal in $G$ and is $>1$ and this contradiction shows that $G$ is simple.

If $P$ and $P^{*}$ are two $S_{p}$ subgroups of $G$ and $P_{0}=P \cap P^{*}>1$, then since $P$ is cyclic, $U=\mathfrak{N}_{G}\left(P_{0}\right) \supseteqq N$ and $U<G$. Since $N$ fails to have a normal $S_{p^{\prime}}$, the same is true of $U$ and thus the $S_{p} P$ of $U$ is normal and $P=P^{*}$. Therefore $P$ is a T. I. set. Now let

$$
S=\mathfrak{C}_{G}(P) \leqq N
$$

If $P^{*}$ is another $S_{p}$ of $G$ and $S^{*}=\sqrt{ }\left(P^{*}\right)$, suppose that $S_{0}=S \cap S^{*}>1$. Now $S_{0}$ is not a $p$-group for otherwise $S_{0} \subseteq P \cap P^{*}=1$, and thus there is some $x \neq 1$ in $S_{0}$ which is a $p^{\prime}$-element. Since

$$
P, P^{*} \cong \mathfrak{F}_{G}(x)<G,
$$

$\mathbb{C}_{G}(x)$ has a normal $S_{p}, L$. Since $x$ is a $p^{\prime}$-element of $N$ we may suppose that $x \in K$ and hence $K \subseteq \mathfrak{E}(x)$ because $K$ is abelian. Thus $K \cong L$ and $K=\mathfrak{N}_{L}(P)$. Since $P$ normalizes $L$, it also normalizes $K$ and this is a contradiction. Therefore $S_{0}=1$ and $S$ is a T. I. set.

Now let $A$ be any maximal $p^{\prime}$-subgroup of $G$ and $B$ a $p^{\prime}$-subgroup with $A \cap B \neq 1$. If $V=\mathfrak{\mho}_{\theta}(A \cap B)<G$ then $A, B \cong V$. If $V$ has a normal $S_{p^{\prime}} L$ then $A \subseteq L$ and by maximality $A=L$ and $B \subseteq A$. If $V$ has a normal $S_{p} P_{0}$ then $V$ has a possibly not normal $S_{p^{\prime}} L$ and since $V$ is solvable, we may suppose that $A \subseteq L$ by P. Hall's theorem. Thus $A=L$ and some conjugate of $B$ is contained in $A$. In this situation, since $A$ normalizes $P_{0}$ and $P$ is a T. I. set we may conclude that $A$ normalizes some $S_{p}$ of $G$.

If $q$ is a prime, $q \| A \mid$, let $Q$ be an $S_{q}$ of $G$ with $Q \cap A \neq 1$. Then some conjugate of $Q$ is $\subseteq A$ and thus $A$ is a Hall subgroup of $G$. If $A^{*}$ is another maximal $p^{\prime}$-subgroup of $G$ with $q \| A^{*} \mid$ then $A^{*}$ meets some conjugate of $A$ and we may conclude that $A^{*}$ is conjugate to $A$ and $|A|=\left|A^{*}\right|$. If $A$ does not normalize an $S_{p}$ of $G$ then $A$ is disjoint from all other maximal $p^{\prime}$-subgroups of $G$ and $A$ is a T. I. set. In this situation let $Q \subseteq A$ be an $S_{q}$ of $G$. Since $A$ is abelian, $Q \triangle \mathfrak{R}_{G}(A)$ and since $A$ is a $T$. I. set, $\mathfrak{N}_{G}(Q)=\mathfrak{R}_{G}(A)$ and thus by Burnside's theorem, $\mathfrak{N}_{G}(A)>A$. By the maximality of $A$ it follows that $p|| \mathfrak{R}(A) \mid$ and some element of order $p$ normalizes $A$.

Continuing with the situation where $A$ does not normalize an $S_{p}$ of $G$, suppose some element $y$ of order $p$ centralizes some $a \neq 1$ in $A$. We may suppose $y \in P$ and since $y \in P^{a}$ also, we conclude that $P=P^{a}$ and we may suppose $a \in K$. Then $K \bigcap A \neq 1$ and therefore $K \subseteq A$. Since $A$ is a $T$. $I$. set, $y$ normalizes $A$ and $K=\Re_{A}(\langle y\rangle)$ and thus $y$ normalizes and hence centralizes $K$ and therefore $K$ centralizes all of $P$ and we have a contradiction. Thus no $a \in A$ different from 
1 commutes with any element of order $p$ and since $A$ is normalized by such an element we have $|A| \equiv 1 \bmod p$.

Let $A_{0}, A_{1}, \cdots, A_{s}$ be a collection of maximal $p^{\prime}$-subgroups of $G$ with all $\left|A_{i}\right|$ distinct and including all posibilities and with $K \cong A_{0}$. If $q|| G \mid$ and $q \neq p$ then some $A_{i}$ contains an $S_{q}$ of $G$ and if $q|| A_{j} \mid$ also, then $A_{j}$ meets some conjugate of $A_{i}$ and as we have seen this implies that $\left|A_{j}\right|=\left|A_{i}\right|$ and thus $j=i$. Therefore

$$
|G|=|P| \prod_{i=0}^{s}\left|A_{i}\right|
$$

Since $K \subseteq A_{0}$, no $A_{i}$ for $i>0$ can normalize an $S_{p}$ of $G$ and if $A_{0}>K$, the same is true of $A_{0}$. In this situation no $p$-element commutes with a $p^{\prime}$-element nontrivially and thus $\mathfrak{c}_{\theta}(P)=P$ and $K$ is isomorphic with a subgroup of the automorphisms of $P$ and since $P$ is cyclic and $p \nmid|K|,|K| \leqq p-1$. Continuing with the assumption that $A_{0}>K$ we see that all $\left|A_{i}\right| \equiv 1 \bmod p$ and thus $|G| /|P| \equiv 1 \bmod p$. By Sylow's theorem, $|G| /|K||P| \equiv 1 \bmod p$ and therefore $1 \equiv|G| /|P| \equiv$ $|K| \bmod p$. Since $|K|<p$ we must have $|K|=1$ and this is a contradiction by Burnside's theorem. Therefore $A_{0}=K$ and $K$ is a maximal $p^{\prime}$-subgroup.

Let $Z=\mathfrak{S}_{K}(P)<K$ and let $Q$ be an $S_{q}$ of $K$. Clearly, $K \subseteq \Re_{G}(Q)$ and thus by Burnside's theorem, $K<\mathfrak{N}_{G}(Q)$ and hence $p|| \mathfrak{N}(Q) \mid$. Since $Z<K$ we may choose $q$ with $Q \nsubseteq Z$. If $\mathfrak{N}(Q)$ has a normal $S_{p} P_{0}$ then $Q$ centralizes $P_{0}$ and therefore $Q$ centralizes all of some $S_{p}$ subgroup of $G$. It follows that $Q$ is contained in some conjugate of $Z$ and thus $Q^{u} \subseteq Z$. However $Q^{u}$ is therefore an $S_{q}$ of the abelian $K$ and $Q^{u}=Q$. This contradicts $Q \nsubseteq Z$ and thus $\mathfrak{R}(Q)$ fails to have a normal $S_{p}$ and hence has a normal $S_{p^{\prime}} L$ and $L \supseteqq K$. By the maximality of $K, K=L$ and $K$ is normalized by an element $x$ of order $p$. If $x \in P^{*}$, an $S_{p}$ of $G$, suppose $K \cong \mathfrak{N}\left(P^{*}\right)$. Then $K \cong \mathfrak{N}(\langle x\rangle)$ and thus $x$ centralizes $K$ and therefore $K$ centralizes all of $P^{*}$. Since $K P^{*}=N_{G}\left(P^{*}\right)$ we have a contradiction and no $S_{p}$ containing $x$ is normalized by $K$. In particular, $x \notin P$. We conclude that each of $P, P^{x}, \cdots, P^{x^{p-1}}$ is normalized by $K$ and they are all distinct. Now $\mathfrak{夭}_{K}\left(P^{x^{i}}\right)=Z^{x^{i}}$ and since $\mathfrak{S}_{G}(P)$ is a $T$. $I$. set $Z^{x^{i}} \cap Z^{x^{j}}=1$ unless $i=j$.

Put $|Z|=c$. Since the direct product $Z \times Z^{x} \subseteq K$ we have $c^{2}|| K \mid$ and we set $|K|=c^{2} t$. We have $\left|K-\bigcup Z^{x^{i}}\right|=c^{2} t-p(c-1)-1$. Now $K / Z$ is a $p^{\prime}$-group isomorphic with a subgroup of the automorphisms of $P$ and thus is cyclic of order dividing $p-1$. Since $[K: Z]=$ $c t$, we have $c t \mid(p-1)$.

If $x$ centralizes any $a \neq 1$ in $K$ then $a$ normalizes and thus centralizes a full $S_{p} P^{*}$ of $G$ with $x \in P^{*}$. If $b \in K$ then $a^{b}=a$ centralizes 
$\left(P^{*}\right)^{b}$ and thus $P^{*}=\left(P^{*}\right)^{b}$ because $\widetilde{C}_{G}\left(P^{*}\right)$ is a $T$. $I$. set and thus $K$ normalizes $P^{*}$. We have seen that this is impossible and thus $x$ acts without nontrivial fixed points on $K$ and $p \mid\left(c^{2} t-1\right)$.

We have then, $p \mid\left(p-1+c^{2} t\right)$ and since $c t \mid(p-1)$,

$$
p \mid\left[\frac{p-1}{c t}+c\right] \text {. }
$$

Since both $p-1 / c t$ and $c$ divide $p-1$, we have $(p-1) / c t+c<2 p$ and thus $(p-1) / c t+c=p$. This implies that $c \mid((p-1) / c t-1)$ and $p-1 / c t \mid(c-1)$. It follows that either $p-1 / c t=1$ or $c=1$. If $c=1$ then $t=1$ and thus $|K|=1$ and this is a contradiction and therefore $p-1 / c t=1$. This yields $t=1$ and $c=p-1$ and thus $|K|=(p-1)^{2}$. We have then $\left|K-\bigcup Z^{x^{i}}\right|=c^{2} t-p(c-1)-1=0$ and thus $K=$ $\cup Z^{x^{i}}$. We may therefore apply Lemma 2 to $K$ and conclude that $K$ is an elementary abelian $q$-group for some prime $q$. Since $K / Z$ is cyclic of order $c t=p-1$, we conclude that $p-1=q$ and thus $p=3$ and $q=2$. Therefore $\left|\mathfrak{N}_{\theta}(P)\right|=|P||K|=4|P|$ and

$$
\left|\mathfrak{\mho}_{G}(P)\right|=|P||Z|=2|P| \text {. }
$$

If $H<G$ has even order then so does an $S_{p^{\prime}}$ of $H$ and thus a maximal $p^{\prime}$-subgroup containing it has even order and this order must equal $\left|A_{0}\right|=|K|=4$ and therefore $|H| \mid(4|P|)$. Since $\mathfrak{\Xi}_{G}(P)$ is a $T$. $I$. set, the proposition applies and $G$ is not simple. This contradiction proves the theorem.

We note here that an alternate method of completing the proof is to use the theorem of Brauer, Suzuki and Wall [2] instead of the proposition given here in $\S 1$. While there are some similarities in the proofs of these two results, the Brauer-Suzuki-Wall theorem is considerably deeper.

3. Here we prove our second theorem.

Theorem II. Let $G$ be a group and let $p \neq 2$ and $q$ be primes dividing $|G|$. Suppose for every $H<G$ which is not a q-group or $a q^{\prime}$-group that $p|| H \mid$. If $q^{a}$ is the q-part of $|G|$ and $p>q^{a}-1$ or if $p=q^{a}-1$ and an $S_{p}$ of $G$ is abelian then no primes but $p$ and $q$ divide $|G|$.

Proof. If the theorem is false, let $G$ be a minimal counter-example. Every $H<G$ which is neither a $q$-group nor a $q^{\prime}$-group satisfies the hypotheses and thus none has order divisible by any prime different from $p$ and $q$. Suppose $N \triangle G$ with $1<N<G$. If $q|| N \mid$ then no other prime but $p$ can also divide it and thus some prime 
$r \neq p, q$ divides $[G: N]$. If $Q$ is an $S_{q}$ of $N$ then $\mathfrak{N}_{G}(Q) N=G$ and since $r \nmid|N|, r|| \mathfrak{N}_{G}(Q) \mid$ and thus $G$ has a subgroup of order $r|Q|$. This contradiction shows that $q \nmid|N|$. If any $r \neq p$ divides $|N|$, let $R$ be an $S_{r}$ of $N$. Then $\mathfrak{N}_{G}(R) N=G$ and since $q \nmid|N|, q|| \mathfrak{N}_{G}(R) \mid$ and $G$ has a subgroup of order $q|R|$. This contradiction shows that $N$ must be a $p$-group.

If $Q$ is any $q$-subgroup of $G$ then $\mathfrak{R}_{G}(Q)<G$ and thus is not divisible by any prime different from $p$ or $q$. If for every $Q>1, \mathfrak{R}_{G}(Q) / \mathfrak{E}_{G}(Q)$ is a $q$-group then by Frobenius' theorm (see for instance 21.8 of [4]) $G$ has a normal $S_{q^{\prime}}$ which must be a $p$-group and this is a contradiction. Thus for some $Q$, an $S_{p}$ of $\mathfrak{N}_{G}(Q)$ fails to centralize $Q$ and in particular is not normal. Thus an $S_{p}$ of $G$ is not normal and $Q$ is normalized by an element $x$ of order $p^{b}$ which does not centralize it. Some orbit of the elements of $Q$ thus has size $\geqq p$ and $q^{a} \geqq|Q| \geqq p+1 \geqq q^{a}$. We have equality and thus $p+1=q^{a}$ and $Q$ is a full $S_{q}$ of $G$, all of whose nonidentity elements are conjugate under $x$. Thus since $p \neq 2, q=2$ and all 2-elements of $G$ are involutions and in one class. Furthermore, by hypothesis, an $S_{p}$ subgroup $P$ of $G$ is abelian.

If $G$ has the proper normal subgroup $N$ then we have seen that $N$ is a $p$-group but since $G$ does not have a normal $S_{p}, p \mid[G: N]$. If $N \subseteq H<G$ and $q \mid[H: N]$ then the only other prime which can divide $[H: N]$ is $p$ and thus $G / N$ satisfies the hypothesis and if $N>1$ we have a contradiction. This shows that $G$ is simple.

If $H<G$ has even order and an $S_{2}$ of $H$ is not normal then $H$ does not have a normal $p$-complement. If $P_{0}$ is an $S_{p}$ of $H$ then by Burnside's theorem, $P_{0}$ is properly contained in its normalizer in $H$. Therefore $\left[H: \mathfrak{N}_{H}\left(P_{0}\right)\right]<\left[H: P_{0}\right] \leqq 2^{a}=p+1$. By Sylow's theorem then, $P_{0} \triangle H$.

Suppose $x \neq 1$ is a real element of $G$. Then $\mathfrak{N}_{G}(\langle x\rangle)<G$ has even order and since the only 2-elements are involutions, the order of $x^{2}$ is a power of $p$ and $x^{2}$ is a real element. If $G$ has no nonidentity real $p$-elements then for every real $x \in G, x^{2}=1$. Since the product of two involutions is real, the set $\left\{x \mid x^{2}=1\right\}$ is a normal subgroup of $G$. Therefore there exists $y \neq 1$, a real $p$-element. Since $y$ is transformed into its inverse by an element of $\mathfrak{N}_{G}(\langle y\rangle), y$ is not central in that group and thus $\mathfrak{N}_{\theta}(\langle y\rangle)$ does not have a normal $S_{2}$. It therefore has a normal $S_{p}$ which is a full $S_{p}$ subgroup, $P$ of $G$ and thus $\mathfrak{R}_{\theta}(P)$ has even order. It follows that $\mathfrak{R}(P)=P S$ where $S$ is contained in an $S_{2} T$ of $G$ and $P$ is the unique $S_{p}$ of $G$ containing $y$.

If no involution centralizes any nonidentity $p$-element then $S$ acts in a Frobenius manner on $P$ and being abelian, it must be cyclic and thus have order 2. If $t \in T$ is an involution then $\mathbb{E}_{G}(t)=T$ and in 
the terminology of Lemma $1, m=|G| / 2^{a}$ and $u=2^{a}-1$. If $1 \neq$ $s \in S$ then $s$ inverts every element of $P$. Therefore each nonidentity element of $P$ is real and thus is contained in a unique $S_{p}$ and hence $P$ is a $T$. I. set. Thus if any two elements of $P$ are conjugate in $G$ they are conjugate in $\mathfrak{N}_{G}(P)$ and thus are inverses and the nonidentity elements of $P \operatorname{span}(|P|-1) / 2$ classes of $G$. These are the only real classes other than $\{1\}$ and the class of involutions and thus in Lemma $1, r=(|P|-1) / 2$. If $x \neq 1, x \in P$ then $\mathfrak{F}_{G}(x)=\mathfrak{F}_{P S}(x)=P$ and the set of involutions transforming $x$ to $x^{-1}$ is the coset Ps. Therefore in Lemma $1, v_{i}=|P|$ and $\left|K_{i}\right|=[G: P]$ for each $i$. The lemma yields

$$
m^{2}=m\left(2^{a}-1\right)+\frac{|P|-1}{2}|P|[G: P] .
$$

Since $|P| \mid m$ and $2^{a}-1=p, p|P|$ divides the left side and the first term on the right side but not the remainder of the right side of the above equation and thus we have a contradiction. Therefore an involution centralizes some element of order $p$.

Now let $C=\mathbb{C}_{G}(T)$ and suppose $C>T$. Then $C=T \times P_{1}$ where $P_{1}>1$ is a $p$-subgroup of $G$. Set $A=\mathbb{\mho}_{G}\left(P_{1}\right) \supseteqq C$. Either $T \triangle A$ or an $S_{p}$ subgroup $P^{*}$ of $A$ (which is a full $S_{p}$ of $G$ ) is normal. If $P^{*} \triangle A$ then since $|A|=|P||T| \geqq\left|\mathfrak{N}_{G}(P)\right|, A=\mathfrak{N}_{G}\left(P^{*}\right)$ and

$$
1 \neq P_{1} \subseteq P^{*} \bigcap 3\left(\Re_{\theta}\left(P^{*}\right)\right)
$$

and this is impossible in a simple group by 13.5.5 of [5]. Thus $T \triangle A$. Let $s \in S, s \neq 1$ and let $B=\mathbb{C}_{G}(s)$. If $P_{2}$ is an $S_{p}$ of $B$ then $s \in \mathfrak{N}_{B}\left(P_{2}\right)$ and thus $\left[B: \mathfrak{R}_{B}\left(P_{2}\right)\right]<p+1$ and $P_{2} \triangle B$. Since $P_{1} \subseteq B$ we have $P_{1} \subseteq P_{2}$ and thus $P_{2} \subseteq A$ and thus $P_{2}$ normalizes $T$. Since $T \cong B, T$ normalizes $P_{2}$ and thus $P_{2}$ centralizes $T$ and $P_{2} \subseteq P_{1}$. Now

$$
\mathfrak{夭}_{P}(s)=P \cap B=P \bigcap P_{2} \subseteq P \bigcap P_{1} \subseteq P \cap 3\left(\Re_{G}(P)\right)=1
$$

and therefore $S$ acts without nontrivial fixed points on $P$ and every $p$-element of $G$ is real. In particular $x \in P_{1}, x \neq 1$ is real. However, we have $\mathfrak{N}_{G}(\langle x\rangle) \supseteqq A$ and since $|A|=|P||T|$, we have equality and $x$ is central in $\mathfrak{R}_{G}(\langle x\rangle)$ and this is a contradiction. We have shown that $C=\mathfrak{\Im}_{G}(T)=T$.

If $x \neq 1$ is a $p$-element centralized by an involution then $\mathfrak{S}_{G}(x)$ has even order but does not contain a full $S_{2}$ of $G$ and thus has a normal $S_{p}$ which is a full $S_{p}$ of $G$. Hence $x$ is contained in a unique $S_{p}$ of $G$ which is normalized by an involution centralizing $x$. By taking conjugates we may suppose that $x \in P$ is centralized by $s \in S$. Put $E=\mathfrak{夭}_{P}(s)>1$. Now $\mathfrak{\Im}_{G}(s)$ has the normal $S_{p} P_{0} \supseteqq E$ and since $E$ can meet no $S_{p}$ of $G$ other than $P$ we see that $P_{0} \subseteq P$ and thus 
$P_{0}=E$. If $P^{*} \neq P$ is an $S_{p}$ of $G$ then $P_{0} \cap P^{*}=1$ and thus $\mathfrak{S}_{P *}(s)=1$.

Choose $t \in S, t \neq 1$. Since all involutions of $T$ are conjugate in $\mathfrak{R}(T)$, choose $u \in \mathfrak{R}(T)$ with $s=t^{u}$. If $P^{u} \neq P$, then $1=\mathfrak{S}_{P^{u}}(s)=$ $\mathfrak{夭}_{P} u\left(t^{u}\right)=\mathfrak{\complement}_{P}(t)^{u}$ and thus $\mathfrak{\Im}_{P}(t)=1$. Otherwise, $P^{u}=P$ and $u \in \mathfrak{R}(P)=$ $P S$ so that $u=r y$ for some $r \in S$ and $y \in P$. Now $S^{u}$ normalizes $P$ and $S^{u} \cong T$ and thus $S^{u} \subseteq \mathfrak{N}_{T}(P)=S$ and therefore $S=S^{u}=S^{y}$ and $y \in \mathfrak{N}_{P}(S)$. This group is normalized and thus centralized by $S$ and $y \in P \cap 3\left(\Re_{\theta}(P)\right)$ which as we have seen is trivial. Thus $y=1$ and $u=r$ and hence $s=t$. We have therefore shown that $s$ is the only involution in $S$ which centralizes any nonidentity element of $P$.

If $|S|=2$ then $1 \neq \mathfrak{S}_{P}(s) \subseteq P \cap 3\left(\mathfrak{N}_{G}(P)\right)$ and this is a contradiction. Thus $|S| \geqq 4$ and we may find two involutions $t$ and $t^{\prime}$ in $S$, both different from $s$. Then both $t$ and $t^{\prime}$ invert every element of $P$. Therefore $t t^{\prime}$ centralizes $P$ and hence $t t^{\prime}=s$ and $\langle s\rangle$ has index 2 in $S$. We have now $\left|\mathfrak{R}_{G}(P)\right|=|S||P|=4|P|$ and $\left|\mathfrak{E}_{G}(P)\right|=$ $|\langle P, s\rangle|=2|P|$. Since we have seen that a nontrivial $p$-element which is centralized by an involution is in only one $S_{p}, P$ is a $T$. $I$. set. If $P^{*} \neq P$ is an $S_{p}$ of $G$ then if $\mathfrak{S}(P) \cap \mathbb{S}\left(P^{*}\right)>1$ it is not a $p$-group and thus contains an involution. Because $P \triangle \mathfrak{F}_{G}(s)$ this is impossible and $\mathfrak{F}_{G}(P)$ is a $T$. $I$. set. Furthermore, since $T \subseteq \mathfrak{F}(s)$, $T$ normalizes $P$ and $T=S$. Therefore $|T|=4=p+1$ and $p=3$. If $H<G$ has even order then $|H| \mid(|T||P|)$ and the hypotheses of the proposition are satisfied. Since $G$ is simple, we have a contradiction and the theorem is proved.

We note that for $p=2$ we can get a counterexample to the theorem by taking $G=A_{5}$ and $q=3$.

\section{REFERENCES}

1. R. Brauer and K. A. Fowler, On groups of even order, Ann. of Math. 62 (1955), $565-583$.

2. R. Brauer, M. Suzuki and G. E. Wall, A characterization of the one-dimensional unimodular projective groups over finite fields, Illinois. J. Math. 2 (1958), 718-745.

3. C. Curtis and I. Reiner, Representation Theory of Finite Groups and Associative Algebras, Interscience, New York, 1962.

4. W. Feit, Characters of finite groups, Mimeographed notes, Yale University Math. Dept., 1965.

5. W. R. Scott, Group Theory, Prentice Hall, Englewood Cliffs, N. J., 1964.

Received August 8, 1966.

UNIVERSITY OF CHICAGO 


\section{PACIFIC JOURNAL OF MATHEMATICS}

\section{EDITORS}

\section{H. ROYDEN \\ Stanford University \\ Stanford, California}

\author{
J. P. JANS \\ University of Washington \\ Seattle, Washington 98105
}

J. DugundJI

Department of Mathematics

Rice University

Houston, Texas 77001

RichaRd ARENS

University of California

Los Angeles, California 90024

\section{ASSOCIATE EDITORS}
E. F. BECKENBACH
B. H. NeumanN
F. WOLF
K. YOSIDA

\section{SUPPORTING INSTITUTIONS}

\author{
UNIVERSITY OF BRITISH COLUMBIA \\ CALIFORNIA INSTITUTE OF TECHNOLOGY \\ UNIVERSITY OF CALIFORNIA \\ MONTANA STATE UNIVERSITY \\ UNIVERSITY OF NEVADA \\ NEW MEXICO STATE UNIVERSITY \\ OREGON STATE UNIVERSITY \\ UNIVERSITY OF OREGON \\ OSAKA UNIVERSITY \\ UNIVERSITY OF SOUTHERN CALIFORNIA
}

\author{
STANFORD UNIVERSITY \\ UNIVERSITY OF TOKYO \\ UNIVERSITY OF UTAH \\ WASHINGTON STATE UNIVERSITY \\ UNIVERSITY OF WASHINGTON

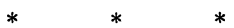 \\ AMERICAN MATHEMATICAL SOCIETY \\ CHEVRON RESEARCH CORPORATION \\ TRW SYSTEMS \\ NAVAL ORDNANCE TEST STATION
}

Mathematical papers intended for publication in the Pacific Journal of Mathematics should be typewritten (double spaced). The first paragraph or two must be capable of being used separately as a synopsis of the entire paper. It should not contain references to the bibliography. Manuscripts may be sent to any one of the four editors. All other communications to the editors should be addressed to the managing editor, Richard Arens at the University of California, Los Angeles, California 90024.

50 reprints per author of each article are furnished free of charge; additional copies may be obtained at cost in multiples of 50 .

The Pacific Journal of Mathematics is published monthly. Effective with Volume 16 the price per volume (3 numbers) is $\$ 8.00$; single issues, $\$ 3.00$. Special price for current issues to individual faculty members of supporting institutions and to individual members of the American Mathematical Society: $\$ 4.00$ per volume; single issues $\$ 1.50$. Back numbers are available.

Subscriptions, orders for back numbers, and changes of address should be sent to Pacific Journal of Mathematics, 103 Highland Boulevard, Berkeley 8, California.

Printed at Kokusai Bunken Insatsusha (International Academic Printing Co., Ltd.), 7-17, Fujimi 2-chome, Chiyoda-ku, Tokyo, Japan.

PUBLISHED BY PACIFIC JOURNAL OF MATHEMATICS, A NON-PROFIT CORPORATION

The Supporting Institutions listed above contribute to the cost of publication of this Journal, but they are not owners or publishers and have no responsibility for its content or policies. 


\section{Pacific Journal of Mathematics}

\section{Vol. 23, No. 2 \\ April, 1967}

Herbert Stanley Bear, Jr. and Bertram John Walsh, Integral kernel for

one-part function spaces .......................... 209

Mario Borelli, Some results on ampleness and divisorial schemes ....... 217

John A. Erdos, Unitary invariants for nests . ................... 229

Nathaniel Grossman, The volume of a totally-geodesic hypersurface in a pinched manifold.................................. 257

D. M. Hyman, A generalization of the Borsuk-Whitehead-Hanner theorem ............................................. 263

I. Martin (Irving) Isaacs, Finite groups with small character degrees and large prime divisors ............................. 273

I. Martin (Irving) Isaacs, Two solvability theorems ................ 281

William Lee Johnson, The characteristic function of a harmonic function in a locally Euclidean space ............................... 291

Ralph David Kopperman, Application of infinitary languages to metric spaces ............................................. 299

John Lauchlin MacDonald, Relative functor representability ............ 311

Mahendra Ganpatrao Nadkarni, A class of measures on the Bohr group.... 321

Keith Lowell Phillips, Hilbert transforms for the p-adic and p-series fields....................................... 329

Norman R. Reilly and Herman Edward Scheiblich, Congruences on regular semigroups ...

Neil William Rickert, Measures whose range is a ball ... . .

Gideon Schwarz, Variations on vector measures

Ronald Cameron Riddell, Spectral concentration for self-adjoint operators. .

Haskell Paul Rosenthal, A characterization of restrictions of

Fourier-Stieltjes transforms ................... 